\title{
Alteraciones hematológicas en pacientes con cáncer: antes, durante y después del tratamiento quimioterapéutico
}

\section{Hematological alterations in cancer patients: before, during and after chemotherapeutic treatment}

\author{
Ruth Miros-Prieto, ${ }^{1 *}$ y Marina Guadarrama-Olhovich ${ }^{2}$
}

Fecha de recepción: 25 de mayo 2021

Fecha de aceptación: 31 de agosto de 2021

\begin{abstract}
Resumen - Las alteraciones hematológicas en los pacientes con cáncer pueden llegar a ser muy particulares y es importante conocerlas para implementar correcciones terapéuticas oportunas. Suelen ser diferentes en cada etapa de la enfermedad y podrían utilizarse como biomarcadores de la respuesta al tratamiento quimioterapéutico. Algunas de dichas alteraciones son de relevancia diagnóstica y pueden derivar en urgencias oncológicas.
\end{abstract}

Palabras clave: Cáncer, alteraciones hematológicas, síndromes paraneoplásicos, quimioterapia.
Abstract - Hematological alterations in cancer patients can be very particular and it is important to know them in order to implement timely therapeutic corrections. They tend to be different at each stage of the disease and could be used as biomarkers of the response to chemotherapy treatment. Some of these alterations are of diagnostic relevance and can lead to in oncology emergencies.

Key words: Cancer, hematological abnormalities, paraneoplastic syndromes, chemotherapy.

\footnotetext{
${ }^{1}$ Departamento de Patología, Facultad de Medicina Veterinaria y Zootecnia, Universidad Nacional Autónoma de México. Ciudad Universitaria, Avenida Universidad núm. 3000, Coyoacán, C.P. 04510, Ciudad de México, México. *Correo electrónico: ruthmiros@gmail.com ORCID: 0000-0001-5663-2857 ${ }^{2}$ Departamento de Patología, Laboratorio de Patología Clínica, Facultad de Medicina Veterinaria y Zootecnia, Universidad Nacional Autónoma de México. ORCID: 0000-0002-3557-0412
} 


\section{Introducción}

as alteraciones hematológicas se presentan frecuentemente en perros y gatos con cáncer, como consecuencia directa del crecimiento neoplásico, de síndromes paraneoplásicos o asociados al tratamiento quimioterapéutico. Estas alteraciones se caracterizan por aumento o disminución en la cantidad de células sanguíneas, así como cambios en la hemostasia (Childress, 2012).

En la práctica clínica de pequeñas especies es importante conocer estas alteraciones, ya que las anomalías hematológicas pueden ser características de tipos específicos de cáncer y su identificación facilita el diagnóstico oportuno; también participan como biomarcadores de la respuesta al tratamiento del cáncer o del estado de remisión en estos pacientes (Childress, 2012). Es importante recordar que, en pequeñas especies, tales cambios se han relacionado a citotoxicidad por quimioterapia y radioterapia, lo que ocasiona desajustes de las distintas líneas celulares (Childress, 2012, Coffee, Roush \& Higginbotham, 2020, Clermont et al., 2011, Harper \& Blackwood, 2017).

A continuación se revisarán brevemente las diferentes alteraciones hematológicas que se han encontrado en perros y gatos con cáncer antes, durante y después del tratamiento.

\section{Línea eritrocítica}

\section{Anemia}

La anemia es particularmente común en pacientes con neoplasias hematopoyéticas (Tasca et al., 2009, Childress, 2012), hemangiosarcomas y sarcomas histiocíticos; sin embargo, puede presentarse en cualquier paciente con cáncer, con o sin tratamiento. Los mecanismos patogénicos relacionados con la anemia incluyen pérdida de sangre, aumento de la destrucción de eritrocitos y disminución en su producción (Mullin \& Clifford, 2019).

\section{Pérdidas sanguíneas en los pacientes con cáncer}

Se observa con mayor frecuencia en hemangiosarcomas, debido al daño a la membrana endotelial, secundario a vasculitis, insuficiencia hepática y deficiencia de sistema reticuloendotelial (Mullin \& Clifford, 2019). También en linfoma esplénico, especialmente el difuso de células $B$ grandes, como consecuencia de la ruptura de la cápsula del bazo, provocando hemorragia y por lo tanto anemia (Weiss \& Wardrop, 2010).

Los síndromes paraneoplásicos también contribuyen a la pérdida de sangre, lo que se ha relacionado especialmente a los efectos sistémicos gastrointestinales ocasionados en mastocitoma, ya que los ejemplares que lo padecen experimentan liberación de histamina por parte de las células neoplásicas, mismas que estimulan a los receptores $\mathrm{H} 2$ gástricos, provocando expedición excesiva de ácido clorhídrico, lo que favorece la formación de úlceras gástricas que terminan en hemorragias gastrointestinales (Blackwood et al., 2012).

\section{Anemia hemolítica inmunomediada (AHIM)}

Este tipo de anemia se ha reconocido como síndrome paraneoplásico en personas, mas no se ha vinculado directamente con pacientes veterinarios con cáncer (Garden et al., 2019). En humanos, las principales causas de AHIM y cáncer se desarrolla en los desórdenes linfoproliferativos, como la leucemia linfocítica crónica de células $B$, que al producir inmunoglobulinas se ha relacionado con autoanticuerpos específicos contra eritrocitos, detectado con antiglobulina directa hasta en $20 \%$ de los pacientes (Hall, Vickers, McLeod E. \& Barker, 2005) (Figura 1). 
Figura 1. Anemia hemolítica inmunomediada. Se muestra aglutinación (flecha negra), objetivo 100x, tinción Wright, esferocitos (flecha roja) y policromasia (flecha azul).

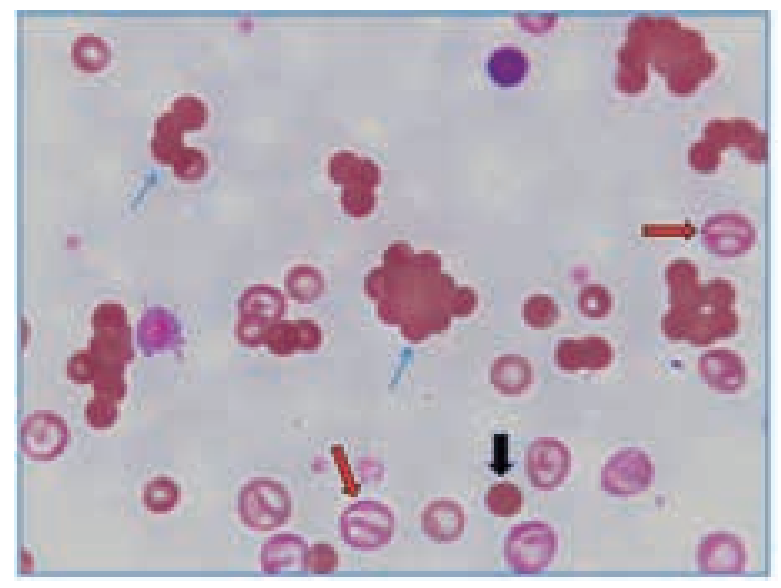

Fuente: Departamento de patología, FMVZ UNAM.

El Colegio Americano de Medicina Interna Veterinaria (ACVIM) hizo una declaración de consenso sobre el diagnóstico de AHIM en perros y gatos, con el que se buscó conocer los valores de la métrica integrada de evidencia (MIE) en pequeñas especies con diferentes neoplasias, según estudios reportados.

Los tipos de cáncer que se incluyeron en perros y gatos fueron leucemia mielocítica o enfermedades mieloproliferativas, leucemia linfocítica, linfoma, sarcoma histiocítico, mieloma múltiple, carcinoma y sarcoma. La MIE tenía una escala de evidencia insignificante y baja, niveles bajos e intermedios e intermedios y altos. El consenso determinó que los pacientes con neoplasias donde se había reportado AHIM tenían un grado de evidencia insignificante, debido a que no hay una aparente asociación y que en la literatura veterinaria faltan vínculos causales entre el cáncer y la AHIM; sin embargo, no puede eliminarse como un posible desencadenante de la enfermedad (Garden et al., 2019).

\section{Disminución en la producción}

La disminución en la producción se puede desarrollar por efectos directos o indirectos sobre la eritropoyesis.
La anemia no regenerativa puede presentarse por remplazo del tejido hematopoyético con tejido neoplásico (mieloptisis), por reducción en la producción o respuesta a la eritopoyetina (EPO) e incluso por inflamación crónica (Tasca et al., 2009).

En un estudio de 210 perros con neoplasias hematopoyéticas, 168/210 (80\%) estaban anémicos, $51 / 210$ (24\%) fueron diagnosticados con leucemia linfoblástica aguda (LLA): 50/51 (98\%) presentaron anemia; a 33/210 (16\%) se les diagnosticó leucemia mieloblástica aguda (LMA): 32/33 (97\%) presentaron anemia; $61 / 210$ (29\%) fueron diagnosticados con leucemia linfocítica crónica (LLC), 46/61 (75\%) presentaron anemia; 65/210 (31\%) fue diagnosticado con linfoma leucémico de alto grado (estadio V); 50/65 (77\%) presentaron anemia. En este tipo de neoplasias hematopoyéticas, la principal anemia es la mieloptisis, por lo que los pacientes son incapaces de producir una o varias líneas celulares (Tasca et al., 2009).

Asimismo, la anemia por disminución en su producción puede estar influenciada por la generación inadecuada de eritropoyetina (EPO) cuando se desarrolla enfermedad renal crónica, o por reducción de la respuesta de las células progenitoras eritrocíticas en la médula ósea a la EPO, disminución de la vida media de eritrocitos en circulación y alteraciones en el metabolismo del hierro (Childress, 2012).

La anemia en los pacientes con cáncer llega a afectar los tiempos de supervivencia, lo que se demostró en un estudio retrospectivo de perros con linfoma y perros con osteosarcoma (Miller et al., 2009), el cual clasificaba a los pacientes como anémicos si tenían un hematocrito $(\mathrm{Ht})<0.40 \mathrm{~L} / \mathrm{L}$. Además, la anemia se subclasificó como moderada ( $\mathrm{Ht}:<0.35 \mathrm{~L} / \mathrm{L}$ ), ligera ( $\mathrm{Ht}: 0.35-0.40 \mathrm{~L} / \mathrm{L})$ y $\sin$ anemia ( $\mathrm{Ht}$ : $>0.40 \mathrm{~L} / \mathrm{L})$. Los perros con linfoma tuvieron un tiempo de vida significativamente menor cuando su hematocrito se encontraba $<0.35 \mathrm{~L} / \mathrm{L}$; aquellos anémicos previos al tratamiento con osteosarcoma tuvieron una mediana de supervivencia más corta que los ejemplares sin anemia y aunque no hubo diferencia estadística significativa en los perros con osteosarcoma 
con y sin anemia, los autores creen que se debió al gran número que murió de manera temprana durante el tratamiento (Miller et al., 2009).

\section{Eritrocitosis}

La eritrocitosis es un hallazgo poco común en pacientes con cáncer. Cuando su origen es primario (médula ósea), recibe el nombre de eritrocitosis absoluta primaria; no obstante, también puede ocurrir como consecuencia de síndromes paraneoplásicos, ya sea en neoplasias renales (linfoma, sarcomas, nefroblastoma) o no renales, como schwannoma y leiomiosarcoma cecal (Taylor et al., 2019, Michael et al., 2019; Hergt et al., 2019; Yamauchi et al., 2004; Sato et al., 2002).

En un análisis de 29 perros con linfoma renal, cuatro de ellos (14\%) tuvieron anemia; sin embargo, 15 (51\%) presentaron eritrocitosis. Los niveles de eritropoyetina (EPO) se midieron en cuatro perros con eritrocitosis; en dos de ellos la concentración de EPO se ubicó dentro de intervalos de referencia y en los otros dos dicha concentración estaba incrementada. Los autores sugirieron que la eritrocitosis pudo estar relacionada a hemoconcentración, pero solo en un perro había incremento de proteínas y posterior a la terapia de líquidos la eritrocitosis persistió, por lo tanto, concluyeron que la eritrocitosis estaba asociada al linfoma renal (Taylor et al., 2019).

La eritrocitosis absoluta primaria está clasificada como síndrome mieloproliferativo crónico en perros que cursan con signos inespecíficos. La enfermedad se caracteriza por la proliferación clonal de células progenitoras eritrocíticas en médula ósea, la cual es independiente de las concentraciones de EPO sérica (Beurlet et al., 2011).

En un estudio de perros con eritrocitosis absoluta primaria se buscó identificar la mutación del gen JAK2, reportado con mutación en $90 \%$ de las personas con este desorden mieloproliferativo. En este estudio no solo se identificó la mutación en JAK2, sino que pudo observarse que en los cinco perros evaluados, las concentraciones de EPO se hallaban por debajo de los intervalos de referencia con eritrocitosis marcada (Beurlet et al., 2011).

Las consecuencias clínicas en estos pacientes con eritrocitosis absoluta primaria son trastornos en la hemostasia, principalmente por síndrome de hiperviscosidad, que puede llegar a concluir en alteraciones neurológicas tales como ataxia, convulsiones, estupor y coma (Accorsi, Passeri, Onofrillo \& lacone, 2005).

\section{Línea plaquetaria y coagulación Trombocitopenia}

Este cambio puede observarse recurrentemente en perros y gatos con cáncer (Finlay, Wyatt \& Black, 2017). Las neoplasias vasculares y hematopoyéticas tienen mayor probabilidad de presentar trombocitopenia (Childress, 2012; Tasca et al., 2009).

Los mecanismos de trombocitopenia en cáncer incluyen disminución en la producción, secuestro, aumento en la destrucción y aumento del consumo o utilización de plaquetas (Finlay et al., 2015).

\section{Disminución en la producción}

La disminución de la producción de plaquetas en la médula ósea frecuentemente se asocia a mieloptisis por neoplasias hematopoyéticas. En el mismo estudio citado anteriormente de 210 perros con neoplasias hematopoyéticas, $110 / 210$ casos (52\%) eran trombocitopénicos, de los cuales 51/210 (24\%) fueron diagnosticados con LLA: 46/51 (90\%) presentaron trombocitopenia. A 33/210 (16\%) se les diagnosticó LMA: 29/33 (88\%) tuvieron trombocitopenia. A 61/210 (29\%) se les encontró LLC: 9/61 (15\%) presentaron trombocitopenia, y a 65/210 (31\%) se les detectó linfoma leucémico de alto grado (estadio V): 26/65 (40\%) presentaron trombocitopenia (Tasca et al., 2009).

La leucemia megacarioblástica aguda con mayor frecuencia produce trombocitopenia, ya que la proliferación neoplásica de precursores plaquetarios en la médula ósea puede asociarse a un estado disfuncional de estas células; si bien pueden ocasionar 
trombocitosis, es común que no haya desprendimiento de plaquetas adecuado a partir del citoplasma de los megacariocitos y por lo tanto se presente justo la disminución de las plaquetas en el torrente sanguíneo. Asimismo, leucemias de origen mielocítico y linfoblásticas agudas pueden cursar con este hallazgo (Comazzi, Gelain, Bonfanti \& Roccabianca, 2010).

Otro mecanismo de reducción de la producción de plaquetas es por el efecto causado por quimioterapia. Se ha observado que perros de razas pequeñas tienen mayor riesgo de mielosupresión motivada por este tipo de fármacos antineoplásicos (Coffee et al., 2020). En un estudio retrospectivo con 101 perros tratados con carboplatino durante tres a cuatro semanas para diferentes neoplasias malignas, 13 de ellos (13\%) tenían pesos <10 kg: 5/13 (38\%) presentó trombocitopenia, ocho (8\%) pesaban entre 10-15 kg: $2 / 8$ (25\%) presentaron trombocitopenia, y 80 perros $(79 \%)$ con pesos $215 \mathrm{~kg}$ : 13/80 (16\%) tenían trombocitopenia, todos grado 3 o 4 a los 14 y 21 días postratamiento, con base en los criterios del Grupo de Oncología Cooperativa Veterinaria para Eventos Adversos (VCOGCTCAE) (Cuadro 1) (Coffee et al., 2020). Con estos datos se concluye que los perros con peso <10 kg tenían mayor riesgo de presentar trombocitopenia posterior al tratamiento con carboplatino.

Cuadro 1. VCOG - CTCAE: criterios del Grupo de Oncología Cooperativa Veterinaria para eventos adversos. LIN: límite inferior normal.

\begin{tabular}{|c|c|c|c|c|c|}
\hline \multirow{2}{*}{$\begin{array}{c}\text { EVENTO } \\
\text { ADVERSO }\end{array}$} & \multicolumn{5}{|c|}{ GRADO } \\
\hline & 1 & 2 & 3 & 4 & 5 \\
\hline 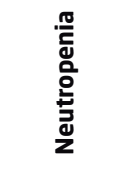 & $1.5 a<\operatorname{LIN}$ & $\begin{array}{l}1.0 \mathrm{a} \\
1.49\end{array}$ & $\begin{array}{l}0.5 a \\
0.49\end{array}$ & $<0.5$ & Muerte \\
\hline 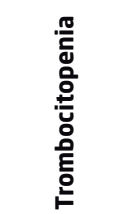 & $100 a<\operatorname{LIN}$ & $\begin{array}{c}50 \mathrm{a} \\
99\end{array}$ & $\begin{array}{c}25 a \\
49\end{array}$ & $<25$ & Muerte \\
\hline
\end{tabular}

Fuente: Modificado de Coffee et al., 2020.

\section{Secuestro por esplenomegalia}

Las neoplasias malignas con esplenomegalia suelen ser el principal origen de trombocitopenia; sin embargo, estas mismas neoplasias pueden cursar con hemoabdomen y la trombocitopenia puede relacionarse tanto con incremento en su consumo como con secuestro, dada la esplenomegalia (Sherwood et al., 2016).

\section{Aumento en el consumo o utilización}

En la mayoría de los casos de pacientes oncológicos con este tipo de trombocitopenia, el aumento en el consumo de las plaquetas se ha relacionado con un estado de hipercoagulabilidad que predispone a los pacientes a desarrollar coagulación intravascular diseminada (CID) (Bruchim, Aroch, Saragusty \& Waner, 2008). Las neoplasias asociadas a CID se abordan más adelante, en los trastornos de la coagulación.

La trombocitopenia grave puede provocar hemorragia potencialmente mortal en el tracto gastrointestinal, en el espacio pulmonar o intracraneal; incluso los episodios hemorrágicos menores, como hemorragias gingivales recurrentes y epistaxis, pueden tener un impacto negativo en la calidad de vida de los pacientes (Finlay et al., 2017).

\section{Trombocitosis}

Las causas generales de trombocitosis en perros y gatos pueden clasificarse en fisiológica, reactiva y esencial. En los pacientes con cáncer la trombocitosis puede ser fisiológica o reactiva (Childress, 2012; Woolcock et al., 2017) (Figura 2). 
Figura 2. Tromobocitosis. Las flechas señalan plaquetas de distintos tamaños y formas. Tinción Wright, 100x.

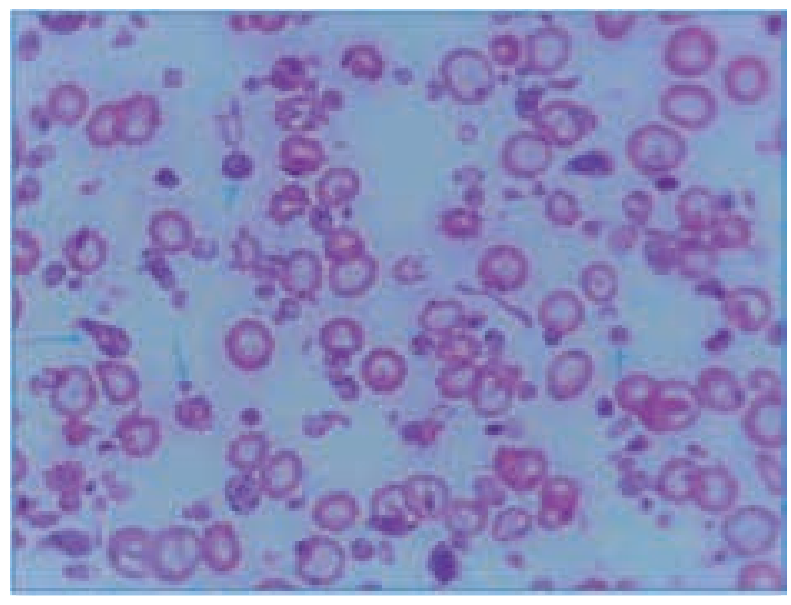

Fuente: Departamento de Patología MVZ UNAM.

En un estudio de 715 perros con trombocitosis, el diagnóstico de neoplasia se identificó en 315 de ellos (44\%). Al evaluar todas las pruebas ( $n=1254), 699$ (55.7\%) se asociaron con un diagnóstico de neoplasia. Los resultados neoplásicos más comunes incluyeron carcinoma urotelial, con $247 / 699$ pruebas (35.3\%), linfoma 190/699 pruebas (27.2\%), mastocitoma $38 / 699$ pruebas $(5.4 \%)$ y hemangiosarcoma $37 / 699$ pruebas (5.3\%). La trombocitosis en estas neoplasias se asoció a trombocitosis reactiva, secundaria a la respuesta inflamatoria en los pacientes. La neoplasia más frecuente fue el carcinoma, que asociado a trombocitosis está documentado en medicina humana. En estos casos la neoplasia produce factor estimulante de colonias granulocíticas-monocíticas (GM-CSF), así como IL-6 y trombopoyetina (TPO). En este estudio no hubo diferencia estadística entre la mediana del recuento de plaquetas en los diagnósticos de carcinoma, sarcoma o tumor de células redondas (Woolcock et al., 2017).

La trombocitosis esencial o primaria está vinculada con la trombocitemia esencial, que es un desorden mieloproliferativo, caracterizado por trombocitosis persistente, consecuencia de la reproducción de células pluripotenciales hematopoyéticas, que conduce a la expansión del compartimento megacariocítico en la médula ósea y a la producción excesiva y autónoma de plaquetas estructural y funcionalmente anormales. El diagnóstico es por exclusión, se debe descartar la trombocitosis reactiva, la cual rara vez tiene conteos plaquetarios por arriba de $1000 \times 10^{9} / \mathrm{L}$ y cuando está presente no es persistente (Mizukoshi et al., 2006).

Trombocitosis $>600 \times 10 \%$ por más de un mes, hiperplasia megacariocítica, niveles normales de TPO y $\sin$ evidencia de displasia, deficiencia de hierro o inflamación subyacente son criterios que sugieren trombocitosis esencial (Douglas \& Wardrop, 2010). Estos criterios se han tomado de medicina humana como complemento en el diagnóstico en medicina veterinaria.

La administración de vincristina se ha vinculado a trombocitosis en pacientes bajo tratamiento quimioterapéutico. Es probable que este fármaco participe en la megacariopoyesis, haciéndola más efectiva (Woolcock et al., 2017; Douglas \& Wardrop, 2010).

\section{Trastornos en la coagulación}

Los pacientes con cáncer pueden cursar estados tanto de hipocoagulabilidad como de hipercoagulabilidad. La primera puede suceder por disminución en el número de plaquetas secundaria o disminución en la producción, generalmente por mieloptisis relacionada a neoplasias hematopoyéticas (Tasca et al., 2009).

La función plaquetaria puede aminorar comúnmente en pacientes con cáncer por paraproteinemia (mieloma múltiple, linfoma y leucemias del linaje de células B) (Accorsi et al., 2005), ya que la hiperglobulinemia (principalmente por IgA e IgM) produce recubrimiento de las células, entre ellas las plaquetas, lo que evita la adhesión endotelial (Childress, 2012; Accorsi et al., 2005).

La formación de trombos como parte de la hipercoagulabilidad se ha relacionado con la 
alteración en el flujo sanguíneo, daño en el endotelio vascular e hipercoagulabilidad de la sangre (Triada de Virchow) (Childress, 2012). El flujo sanguíneo puede verse afectado por hiperviscosidad o compresión de vasos sanguíneos, produciendo daño endotelial. También el endotelio puede lesionarse directamente por la presencia de células neoplásicas, mismas que tienen la capacidad de producir citocinas inflamatorias (IL- 1, TNF- $\alpha$ ) y que desprenden micropartículas que contienen factor tisular en sus membranas, haciendo que las células endoteliales pierdan sus propiedades antitrombóticas, desencadenando así alteraciones protrombóticas (Bruchim et al., 2008).

La coagulación intravascular diseminada (CID) es una complicación grave en pacientes con distintas neoplasias. En ella, la generación de trombina es excesiva y no controlada; esta generación se produce, amplifica y expande sobre las superficies de las células endoteliales (Stokol, 2012). Las neoplasias que pueden favorecer CID son carcinoma de glándula mamaria, neoplasias mieloproliferativas, linfoma, adenocarcinoma pulmonar y hemangiosarcoma (Bruchim et al., 2008).

En condiciones normales, las plaquetas activadas son la única fuente de fosfatidilserina, pero en CID, los monocitos expresan al factor tisular, las células neoplásicas y las células apoptósicas (Stokol, 2012), ocasionando un estado procoagulante.

\section{Línea leucocitaria Leucopenia}

La causa más común de neutropenia en pacientes oncológicos es causada por la quimioterapia y la radioterapia; puede presentarse otro origen, como disminución en la producción (mieloptisis) o incremento en su consumo en procesos inflamatorios agudos o no controlados (Coffee et al., 2020). La quimioterapia y el bajo peso corporal son factores de riesgo que favorecen a la neutropenia, esto se demostró en el mismo grupo de 101 perros tratados con carboplatino durante tres a cuatro semanas para diferentes neoplasias malignas, 13 perros (13\%) tenían pesos <10 kg: 8/13 (61\%) presentó neutropenia, ocho (8\%) pesaban entre $10-15 \mathrm{~kg}$ : 3/8 (38\%) padecía neutropenia, y 80 perros (79\%) con pesos 215 kg: 14/80 (17\%) presentó neutropenia, todos grado 3 o 4 a los 14 y 21 días postratamiento, con base en los criterios del VCOG-CTCAE (Cuadro 1) (Cofee et al., 2020). Concluyendo que perros con peso $<10 \mathrm{~kg}$ tienen mayor probabilidad de padecer neutropenia posterior al tratamiento con este fármaco.

En 292 registros oncológicos de pacientes tratados con diferentes quimioterapéuticos como lomustina 46/292 (16\%), carboplatino 58/292 (20\%), vincristina 43/292 (15\%), doxorrubicina 54/292 (18\%), ciclofosfamida 47/292 (16\%), mitoxantrona 22/292 (8\%) y vinblastina 22/292 (7\%), se observó neutropenia en 63/292 (22\%). La gravedad de la neutropenia incluyó grado 1 en 5/63 (8\%), grado 2 en $37 / 63$ (59\%), grado 3 en 20/63 (32\%) y grado 5 en $1 / 63(1 \%)$ con base en el VCOGCTCAE (Cuadro 1). Aunque no hubo diferencias estadísticas significativas al compararse la presencia de neutropenia y agentes quimioterapéuticos, se identificó que la responsable de la mayoría de los casos con neutropenia fue la lomustina, seguida del carboplatino (Dos Santos Cunha et al., 2017).

Los factores de riesgo para que los perros desarrollen neutropenia tras la administración de quimioterapia incluyen el bajo peso corporal, como se mencionó anteriormente; no obstante, dado el mecanismo de acción de los quimioterapéuticos, prácticamente cualquier paciente que esté bajo estos esquemas podría desarrollarla (Tumielewicz et al., 2019).

El tratamiento quimioterapéutico debe retrasarse si el recuento de neutrófilos es de 1.5-2.0 ×10\%/L. Una emergencia clínica en los pacientes neutropénicos con signos como hiporexia y letargo puede surgir con un valor de neutrófilos que se encuentre en el nadir 
de neutropenia o próximo a él, principalmente cuando un fármaco quimioterapéutico fue administrado recientemente. El nadir de neutropenia se presenta alrededor de los siete a 10 días luego de la aplicación de la quimioterapia. No es común ver signos clínicos relacionados con la neutropenia, a menos que ésta sea $<1 \times 10^{9} /$ L (Tumielewicz et al., 2019) (Gráfica 1). En este punto se puede favorecer el desarrollo de infecciones bacterianas oportunistas, especialmente si hay fiebre concurrente $\left(>39.2^{\circ} \mathrm{C}\right.$ ) (Tumielewicz et al., 2019).

Los pacientes con infecciones bacterianas graves pueden presentar síndrome de respuesta inflamatoria sistémica (SIRS) o entrar en un estado de sepsis como resultado de la neutropenia (Tumielewicz et al., 2019).

Gráfica 1. Grados de neutropenia con base en VCOGCTCAE y nadir



Fuente: Tumielewicz et al., 2019.

La leucopenia por neutropenia asociada a mieloptisis también puede ocurrir en los pacientes oncológicos. Esta alteración se observó en el estudio de los 210 perros con neoplasias hematopoyéticas, donde 68/210 (32\%) eran neutropénicos, de los cuales 51/210 (24\%) fueron diagnosticados con LLA: 40/51 (78\%) presentaron neutropenia, a 33/210 (16\%) se les diagnosticó con LMA: 21/33 (64\%) presentaron neutropenia, 65/210 (31\%) se encontraron con linfoma leucémico de alto grado (estadio V): 7/65 (11\%) presentaron neutropenia. En este estudio no se menciona la fase de la enfermedad (leucémica o aleucémica), sin embargo, con base en los criterios de exclusión se sugiere que se encontraban en etapa leucémica, donde las leucemias agudas -al ser de origen primario (médula ósea)- tienen mayor prevalencia de cursar con citopenias periféricas por mieloptisis, por efecto de las células neoplásicas sobre las líneas celulares normales. Debido a esto es que hubo diferencias estadísticamente significativas en los perros con leucemias agudas, leucemia linfocítica crónica y con linfoma leucémico de alto grado (estadio V) (Tasca et al., 2009).

\section{Leucocitosis}

Los mecanismos de la leucocitosis neutrofílica mayormente desarrollados en los pacientes son inflamación, estrés y efecto de catecolaminas (Schultze, 2010) (Figura 3).

En algunas neoplasias se han observado leucocitosis extremas que se caracterizan por neutrofilia $>50 \times 10^{9} / \mathrm{L}$ (3.0-11.5 x10\%/L), con desviación a la izquierda marcada (mielocitos o promielocitos) (Schultze, 2010).

Figura 3. Leucocitos, objetivo 40x, tinción Wright. Vista panorámica de frotis sanguíneo de paciente con inflamación

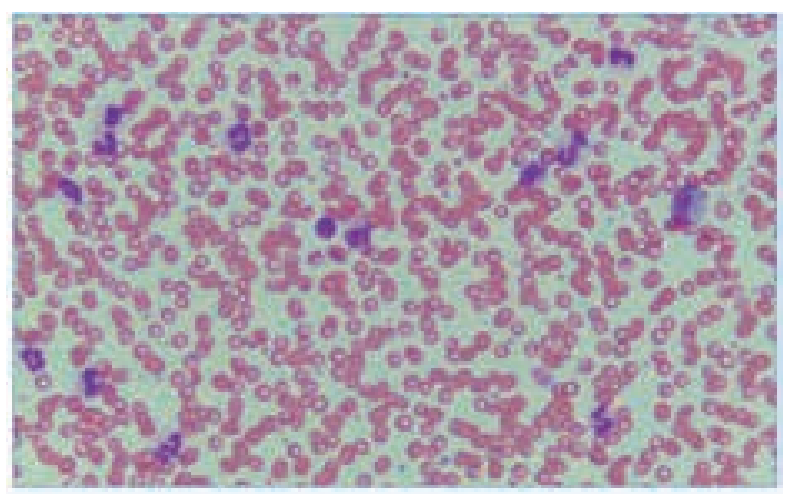

Fuente: Departamento de Patología FMVZ UNAM.

En un estudio de 182 perros con leucocitosis 235 $\mathrm{x} 10^{9} / \mathrm{L}$, las neoplasias mayormente identificadas 
fueron carcinoma renal, hemangiosarcoma, linfoma, mastocitoma, leucemia linfocítica, carcinoma ovárico, mesotelioma, timoma, adenocarcinoma pulmonar, tumor de células de Leydig (Weltan, Leisewitz \& Goddard, 2008). En pacientes con neoplasias, la leucocitosis extrema se atribuye a la producción ectópica del GMCSF o del factor estimulante de colonias granulocíticas (G-CSF) por las células neoplásicas. El GM-CSF incentiva la proliferación de precursores hematopoyéticos para los linajes de neutrófilos, monocitos y eosinófilos, explicando la monocitosis y eosinofilia que también pueden acompañar estas reacciones extremas (Childress, 2012; Petterino et al., 2011).

Otra causa de leucocitosis es la leucemia mielógena crónica (LMC), la cual es resultado de la transformación neoplásica de una célula progenitora. Esta enfermedad deriva de desorden mieloproliferativo, rara en los perros (Tarrant et al., 2001), que a diferencia de la leucocitosis extrema, aquí se presenta desviación a la izquierda desordenada y con morfología atípica de granulocitos en la evaluación de la médula ósea (Childress, 2012; Tarrant et al., 2001).

En ocasiones puede haber leucocitosis por linfocitosis, principalmente en pacientes con linfoma estadio $V$, leucemia linfoblástica aguda (LLA) y leucemia linfocítica crónica (CLL) (Zandvliet, 2016; Tomiyasu et al., 2018; Bromberek et al., 2016) (Figura 4).

Figura 4. Linfocitos en un perro con LLC. Wright 400x

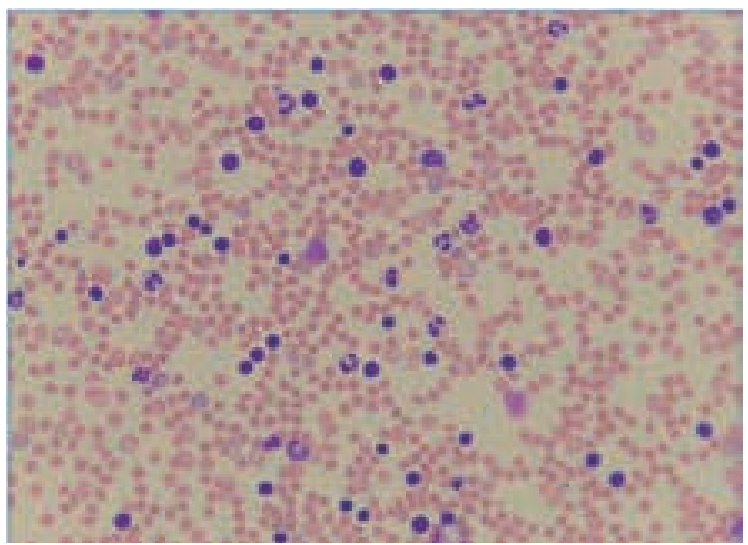

Fuente: Departamento de Patología FMVZ UNAM.

\section{Conclusión}

Los cambios hematológicos en los pacientes con cáncer pueden estar relacionados de manera directa con la multiplicación de células neoplásicas - ser secundarios a síndromes paraneoplásicos, al tratamiento quimioterapéutico o al curso de la enfermedad. Conocer estos hallazgos ayudará a diagnosticarlos oportunamente, determinar el grado de respuesta hacia un tratamiento específico y el acercamiento certero hacia el pronóstico en cada paciente.

\section{Referencias}

Accorsi, P., Passeri, C., Onofrillo, D. \& lacone, A. (2005). Hyperviscosity syndrome in hematological diseases and therapeutic apheresis. The International journal of artificial organs, 28(10), 1032-1038.

Beurlet, S., Krief, P., Sansonetti, A., Briend-Marchal, A., Kiladjian, J. J., Padua, R. A., ... \& Cassinat, B. (2011). Identification of JAK2 mutations in canine primary polycythemia. Experimental hematology, 39(5), 542-545.

Blackwood, L., Murphy, S., Buracco, P., De Vos, J. P., De Fornel-Thibaud, P., Hirschberger, J., Kessler, M., Pastor, J., Ponce, F., Savary-Bataille, K. \& Argyle, D. J. (2012). European consensus document on mast cell tumours in dogs and cats. Veterinary and comparative oncology, 10(3), e1-e29.

Bromberek, J. L., Rout, E. D., Agnew, M. R., Yoshimoto, J., Morley, P. S. \& Avery, A. C. (2016). Breed distribution and clinical characteristics of $B$ cell chronic lymphocytic leukemia in dogs. Journal of veterinary internal medicine, 30(1), 215-222.

Bruchim, Y., Aroch, I., Saragusty, J. \& Waner, T. (2008). Disseminated intravascular coagulation. Compendium, 3(10), 1-16. 
Childress, M. O. (2012). Hematologic abnormalities in the small animal cancer patient. Veterinary Clinics: Small Animal Practice, 42(1), 123-155.

Clermont, T., Leblanc, A. K., Adams, W. H., Leblanc, C. J. \& Bartges, J. W. (2012). Radiotherapy-induced myelosuppression in dogs: 103 cases (2002-2006). Veterinary and comparative oncology, 10(1), 24-32.

Coffee, C., Roush, J. K. \& Higginbotham, M. L. (2020). Carboplatin-induced myelosuppression as related to body weight in dogs. Veterinary and comparative oncology, 18(4), 804-810.

Comazzi, S., Gelain, M. E., Bonfanti, U. \& Roccabianca, P. (2010). Acute megakaryoblastic leukemia in dogs: a report of three cases and review of the literature. Journal of the American Animal Hospital Association, 46(5), 327-335.

Dos Santos Cunha, S. C., Silva, F. B. F., Corgozinho, K. B., Da Silva, K. G. C. \& Ferreira, A. M. R. (2017). Adverse Effects of Chemotherapy in Dogs. World, $7(3), 74-82$.

Finlay, J., Wyatt, K. \& Black, M. (2017). Evaluation of the risks of chemotherapy in dogs with thrombocytopenia. Veterinary and comparative oncology, 15(1), 151-162.

Garden, O. A., Kidd, L., Mexas, A. M., Chang, Y. M., Jeffery, U., Blois, S. L., ... \& Szladovits, B. (2019). ACVIM consensus statement on the diagnosis of immune-mediated hemolytic anemia in dogs and cats. Journal of veterinary internal medicine, 33(2), 313-334. doi: 10.1111/jvim.15441

Hall, A. M., Vickers, M. A., McLeod, E. \& Barker, R. N. (2005). Rh autoantigen presentation to helper $\mathrm{T}$ cells in chronic lymphocytic leukemia by malignant B cells. Blood, 105(5), 2007-2015.
Harper, A. \& Blackwood, L. (2017). Toxicity and response in cats with neoplasia treated with toceranib phosphate. Journal of feline medicine and surgery, 19(6), 619-623.

Hergt, F., Mortier, F., Werres, C., Flatz, K. \& von Bomhard, $W$. (2019). Renal nephroblastoma in a 17-month-old jack Russell terrier. Journal of the American Animal Hospital Association, 55(5), e55503.

Michael, A. E., Grimes, J. A., Volstad, N. J., Osekavage, K. E. \& Koenig, A. (2019). Inappropriate secondary erythrocytosis in a dog with renal sarcoma. Topics in companion animal medicine, 36, 9-11.

Miller, A. G., Morley, P. S., Rao, S., Avery, A. C., Lana, S. E. \& Olver, C. S. (2009). Anemia is associated with decreased survival time in dogs with lymphoma. Journal of veterinary internal medicine, 23(1), 116122.

Mizukoshi, T., Fujino, Y., Yasukawa, K., Matumoto, H., Matsumura, S., Nagasaki, T., ... \& Shimoda, T. (2006). Essential thrombocythemia in a dog. Journal of veterinary medical science, 68(11), 1203-1206.

Mullin, C. \& Clifford, C. A. (2019). Histiocytic sarcoma and hemangiosarcoma update. Veterinary Clinics: Small Animal Practice, 49(5), 855-879.

Petterino, C., Luzio, E., Baracchini, L., Ferrari, A. \& Ratto, A. (2011). Paraneoplastic leukocytosis in a dog with a renal carcinoma. Veterinary clinical pathology, 40(1), 89-94.

Sato, K., Hikasa, Y., Morita, T., Shimada, A., Ozaki, K. \& Kagota, K. (2002). Secondary erythrocytosis associated with high plasma erythropoietin concentrations in a dog with cecal leiomyosarcoma. Journal of the American Veterinary Medical Association, 220(4), 486-490. 
Schultze, A. E. (2010). Interpretation of canine leukocyte responses. Schalm's veterinary hematology (pp. 321-334). Ames, lowa: Wiley-Blackwell.

Sherwood, J. M., Haynes, A. M., Klocke, E., Higginbotham, M. L., Thomson, E. M., Weng, H. Y. \& Towle Millard, H. A. (2016). Occurrence and clinicopathologic features of splenic neoplasia based on body weight: 325 Dogs (2003-2013). Journal of the American Animal Hospital Association, 52(4), 220226.

Stokol, T. (2012). Laboratory diagnosis of disseminated intravascular coagulation in dogs and cats: the past, the present, and the future. Veterinary Clinics: Small Animal Practice, 42(1), 189-202.

Tarrant, J. M., Stokol, T., Blue, J. T., McDonough, S. P. \& Farrell, P. (2001). Diagnosis of chronic myelogenous leukemia in a dog using morphologic, cytochemical, and flow cytometric techniques. Veterinary clinical pathology, 30(1), 19-24.

Tasca, S., Carli, E., Caldin, M., Menegazzo, L., Furlanello, T. \& Gallego, L. S. (2009). Hematologic abnormalities and flow cytometric immunophenotyping results in dogs with hematopoietic neoplasia: 210 cases (2002-2006). Veterinary clinical pathology, $38(1), 2-12$.

Taylor, A., Finotello, R., Vilar-Saavedra, P., Couto, C. G., Benigni, L. \& Lara-Garcia, A. (2019). Clinical characteristics and outcome of dogs with presumed primary renal Iymphoma. Journal of Small Animal Practice, 60(11), 663-670.

Tomiyasu, H., Doi, A., Chambers, J. K., Goto-Koshino, Y., Ohmi, A., Ohno, K. \& Tsujimoto, H. (2018). Clinical and clinicopathological characteristics of acute lymphoblastic leukaemia in six cats. Journal of Small Animal Practice, 59(12), 742-746.
Tumielewicz, K. L., Hudak, D., Kim, J., Hunley, D. W. \& Murphy, L. A. (2019). Review of oncological emergencies in small animal patients. Veterinary medicine and science, 5(3), 271-296.

Weiss, D. J. \& Wardrop, K. J. (eds.). (2010). Schalm's veterinary hematology. John Wiley \& Sons.

Weltan, S. M., Leisewitz, A. L. \& Goddard, A. (2008). A case-controlled retrospective study of the causes and implications of moderate to severe leukocytosis in dogs in South Africa. Veterinary clinical pathology, 37(2), 164-172.

Woolcock, A. D., Keenan, A., Cheung, C., Christian, J. A. \& Moore, G. E. (2017). Thrombocytosis in 715 Dogs (2011-2015). Journal of veterinary internal medicine, 31(6), 1691-1699.

Yamauchi, A., Ohta, T., Okada, T., Mochizuki, M., Nishimura, R., Matsunaga, S., ... \& Sasaki, N. (2004). Secondary erythrocytosis associated with schwannoma in a dog. Journal of veterinary medical science, 66(12), 1605-1608.

Zandvliet, M. (2016). Canine Iymphoma: a review. Veterinary Quarterly, 36(2), 76-104. 